\title{
Desfibrado de maderas obtenidas desde las oficinas salitreras de Humberstone y Santa Laura en Chile. Un sitio del patrimonio mundial
}

\author{
Defibration wood obtained from the Saltpeter Works Humberstone \\ and Santa Laura in Chile. World Heritage Sites
}

\author{
Rodrigo Ortiz ${ }^{*}$ y Robert Blanchette ${ }^{2}$
}

1 Laboratorio de Biodeterioro y Biodegradación de Materiales, Facultad de Ingeniería, Escuela de Construcción Civil, Universidad de Valparaíso, Chile.

\author{
2 Department of Plant Pathology. University of Min- * Autor para correspondencia: rodrigo.ortiz@uuv.cl \\ nesota, EUA.
}

\section{RESUMEN}

En las oficinas salitreras de Humberstone y Santa Laura, declaradas Patrimonio de la Humanidad el año 2005, se presenta un inusual proceso de deterioro en el que es posible observar fibras individuales en la superficie de la madera. En este trabajo se hizo una descripción y evaluación de los daños observados en las instalaciones pertenecientes a ambas oficinas. Así mismo, se complementó el estudio mediante el análisis de las muestras recolectadas a través microscopía electrónica de barrido (SEM). Se realizaron estudios adicionales en las maderas, mediante la evaluación de la velocidad de ultrasonido y extracción de tornillo. Los resultados obtenidos determinaron que el deterioro evidenciado es de tipo químico producto de la acumulación de sales en las maderas, y cuyo efecto está asociado con la degradación de la lamela media altamente lignificada en maderas de las especies de Pseudotsuga menziesii y Nothofagus obliqua. Además, los resultados determinaron que la madera no afectada por la degradación de la sal mantiene sus propiedades mecánicas inalteradas. El deterioro evidenciado, denominado desfibrado, se presenta en mayor o menor medida en la totalidad de las instalaciones. Sin embargo, el mayor daño se encontró en los pisos superiores de la torre de lixiviación de Santa Laura, la que requiere de la aplicación de medidas preventivas asociadas con la eliminación de la sal presente en la superficie del material.

PALABRAS CLAVE: deterioro, evaluación no destructiva, lamela media, microscopía, propiedades mecánicas, sales.

\section{ABSTRACT}

In the Saltpeter Works Humberstone and Santa Laura, declared World Heritage Site in 2005, an unusual deterioration process of the wood takes place in which individual fibers can be observed on the surface of the wood. This paper gives a description and assessment of the damage observed in the installations belonging to both office buildings. Also, the study was supplemented by analysis of samples collected through scanning electron microscopy (SEM). Additional studies in the wood by evaluating the ultrasound velocity and screw extraction were conducted. The results determined that the deterioration is chemical attack due to the accumulation of salts present in the wood, and whose effect is associated with the degradation of the highly lignified middle lamella in Pseudotsuga menziesii and Nothofagus obliqua woods. Moreover, the results showed that the wood unaffected by the degradation of the salt maintains unchanged its mechanical properties. The evident deterioration, which is called defribration, has been developed in varying degrees in all installations. However, the biggest damage is presented in the upper floors of the leaching at tower Santa Laura, which requires the application of preventive measures associated with the removal of salt on the surface of the material.

KEY WORDS: deterioration, nondestructive evaluation, middle lamella, microscopy, mechanical properties, salts. 


\section{INTRODUCCIÓN}

El desarrollo minero de Chile es propio de la "República" (Vial, 1987), concentrándose mayoritariamente en las provincias del norte, en cuyas zonas desérticas existe poca actividad ligada a la agricultura y silvicultura (Culverwell, 2000).

La explotación minera de salitre, que es utilizado como abono agrícola y materia prima de explosivos, convirtió a Chile a fines de la Guerra del Pacífico (1879-1883) en el único fabricante y exportador en el mundo (Vial, 1987), transformándose en el producto principal de la minería hasta la gran depresión de 1930, tiempo en el que se produjo la caída del mercado de consumo debido al desarrollo de métodos alternativos para la síntesis de nitrógeno y la disminución de la demanda de nitratos (Culverwell, 2000). Sin embargo, la explotación histórica del salitre abarca desde la época prehispánica hasta la actualidad (Minvu, 2003).

Las oficinas Salitreras de Humberstone y Santa Laura se encuentran ubicadas en la Región de Tarapacá, Provincia de Iquique, en la denominada Pampa del Tamarugal a $1050 \mathrm{msnm}$. De acuerdo con lo establecido por el Minvu (2003), estos sitios constituyen un conjunto industrial integral, lo que queda de manifiesto debido a que Santa Laura mantiene los rasgos esenciales del procesamiento del salitre en el área industrial, mientras que Humberstone ha mantenido el campamento urbano. Las instalaciones de ambas salitreras conservan ineludibles testimonios de su materialidad, estando constituidas, mayoritariamente, por cubiertas de zinc acanalado, concreto pampino, y madera de Pseudotsuga menziesii (Pino Oregón).

El estado de conservación de los inmuebles presenta evidentes daños provocados por numerosos saqueos, los que en algunos casos han comprometido estructuralmente las instalaciones. Así mismo es posible observar deterioros menores provocados por hongos e insectos. Sin embargo, y durante muchos años, producto del proceso industrial, condiciones climáticas y acumulación de minerales salinos, las maderas de estas salitreras han quedado expuestas a un inusual y lento deterioro no biológico denominado desfibrado, deterioro en el que es posible observar masas de fibras desprendidas de la madera. Si bien es cierto que este proceso de deterioro tiene gran repercusión, los antecedentes bibliográficos que lo estudian y/o describen son escasos (Parameswaran, 1981; Bootle, 2005; Wilkins y Simpson, 1988; Wilcox et al., 1991; Johnson et al., 1992; Schneider et al., 1997; Ku erová et al., 2008)

\section{OBJETIVo}

Considerando lo anterior, y en virtud que las oficinas salitreras de Humberstone y Santa Laura están constituidas de manera importante por instalaciones construidas en madera, se desarrolló el presente trabajo cuyo objetivo fue describir el evidente daño, denominado desfibrado, asociado a condiciones de salinidad al que está sometido este sitio del Patrimonio Mundial.

\section{MATERIALES Y MÉTODOS}

\section{Extracción y almacenamiento de madera}

La madera se extrajo desde las oficinas salitreras de Humberstone y Santa Laura en la provincia de Iquique en Chile. Se consideró una recolección de tipo oportunista. Se recolectaron muestras de madera con diferentes estados de deterioro. Adicionalmente, se recolectaron maderas sin deterioro para la identificación de las especies utilizadas en las estructuras. Las muestras obtenidas se colocaron en bolsas plásticas estériles y se llevaron al Laboratorio de Biodeterioro y Biodegradación de Materiales de la Universidad de Valparaíso donde se almacenaron a $4{ }^{\circ} \mathrm{C}$.

\section{Evaluaciones macroscópicas y microscópicas}

Se realizaron evaluaciones macroscópicas y microscópicas para la identificación de las especies y la determinación de los daños presentes en el material. Las probetas destinadas al análisis macroscópico se prepararon de acuerdo con el protocolo establecido por Díaz-Vaz (1979). Las muestras para microscopía electrónica de barrido (SEM) se prepararon utilizando las técnicas previamente descritas por Blanchette y Simpson (1992). La observación microscópica y captura fotográfica se realizó mediante la utiliza- 
ción de un microscopio electrónico de barrido Carl Zeiss, modelo EVO-MA 10.

La identificación macroscópica y microscópica de las especies de madera se realizó, mediante el uso de claves, según lo informado por Díaz-Vaz (1979).

\section{Evaluaciones no destructivas}

Se realizaron evaluaciones, de la capacidad resistente en madera de Pseudotsuga menziesii debido a su utilización mayoritaria como material estructural, mediante la técnica no destructiva de ultrasonido y la técnica pseudo no destructiva de extracción de tornillo. En ambas evaluaciones se utilizaron probetas cúbicas, de 5 centímetros de arista, acondicionadas a $12 \%$ de contenido de humedad.
Para la evaluación mediante ultrasonido se utilizó un equipo, de baja frecuencia con tranductores de polaridad longitudinal de $54 \mathrm{Khz}$, James Instruments Inc. modelo V-meter MKIV. Las mediciones se realizaron en forma directa y en el plano longitudinal a la fibra.

La evaluación mediante extracción de tornillo se realizó utilizando un equipo Fakopp Enterprise de $5 \mathrm{kN}$ de capacidad. Se utilizaron tornillos de $4 \mathrm{~mm}$ de diámetro, los que fueron introducidos en su parte inferior y media con la finalidad de producir una fuerza de arranque en las fibras situadas a una profundidad de entre $20 \mathrm{~mm}$ y 38 $\mathrm{mm}$. Los tornillos se introdujeron en forma perpendicular a la fibra. Se determinó la densidad de la madera de acuerdo con lo señalado por Basterra (2009).
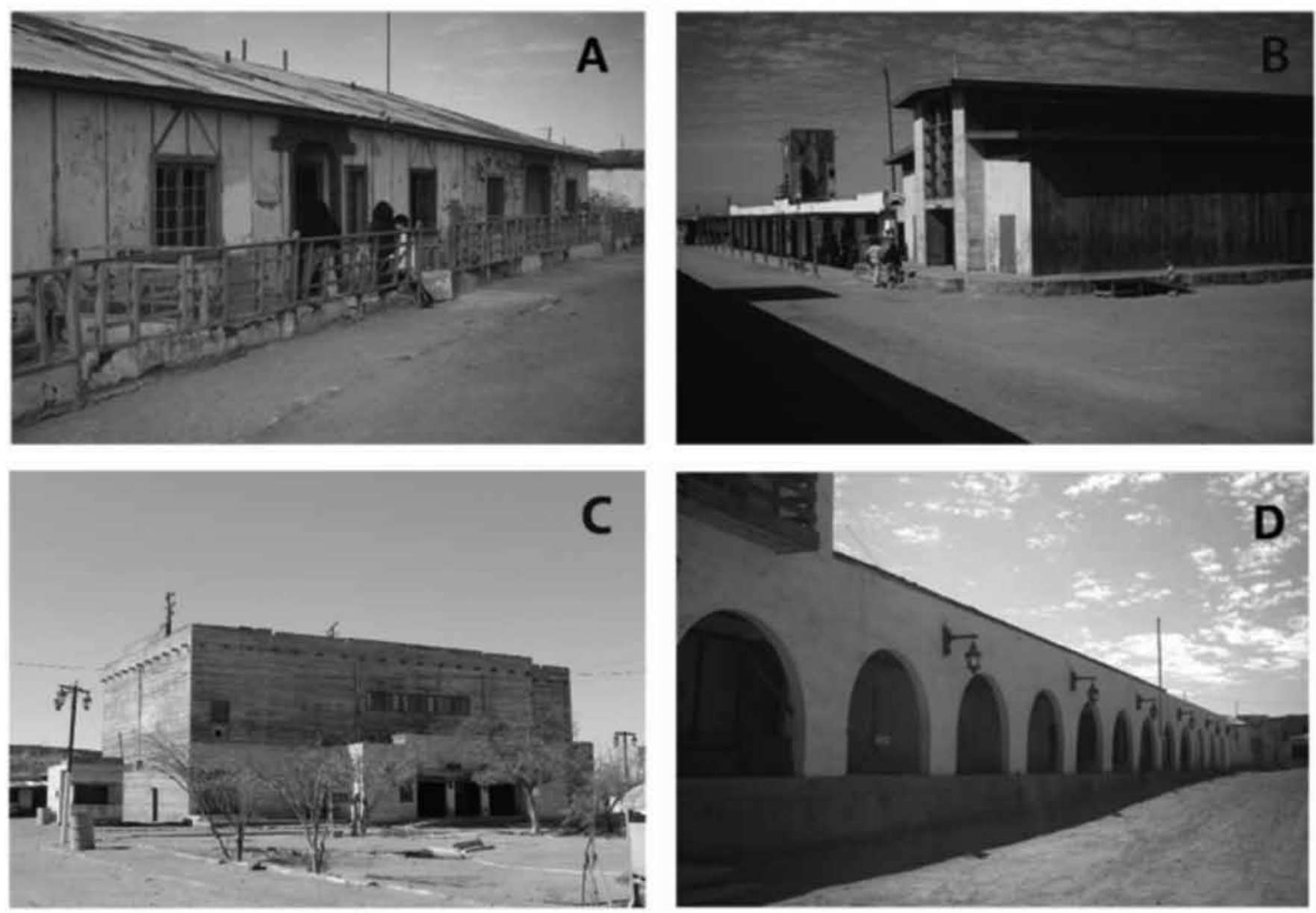

Figura 1. Oficina salitrera Santiago Humberstone. A: Viviendas continuas con Porche. B: Iglesia y Mercado. C: Teatro. D: Pulpería. 


\section{Resultados}

De las oficinas salitreras de Humberstone y Santa Laura se retiraron alrededor de 150 y 100 muestras de madera respectivamente, correspondientes a las zonas: Industrial, Edificios y Espacios Públicos y Campamento (Figs. 1, 2 y 3). La identificación macro y microscópica de las muestras de maderas analizadas correspondieron a las especies Nothofagus obliqua y Pseudotsuga menziesii, siendo esta última la especie de mayor uso.

\section{Oficina Salitrera Santiago Humberstone}

\section{Zonas de edificios, espacios públicos y campamento}

En general las maderas presentes en ambas zonas (Figs. $1 \mathrm{~A}, 1 \mathrm{~B}, 1 \mathrm{C}$ y $1 \mathrm{D})$ se encuentran relativamente sanas, lo
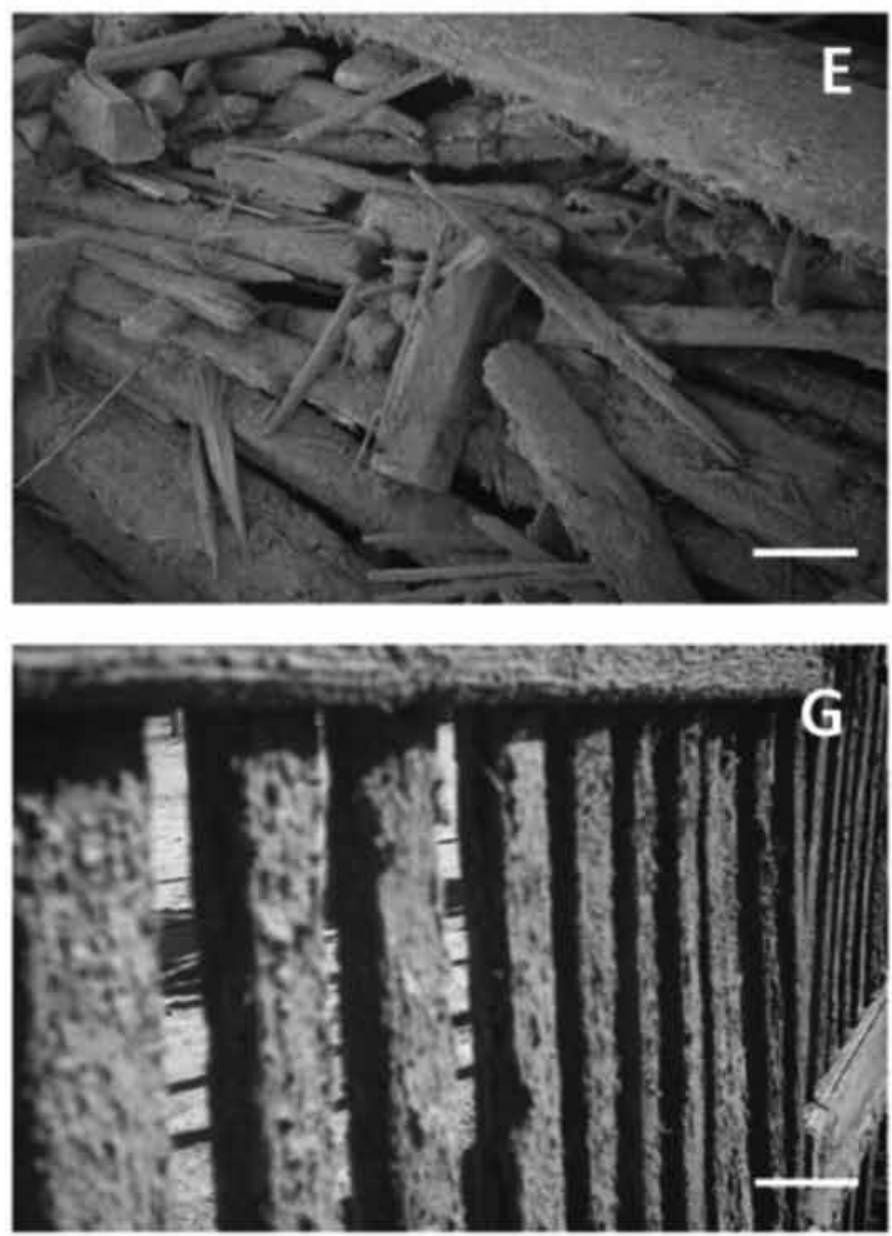

que ha permitido que parte importante de las edificaciones que las constituyen sean accesibles y sin riesgo para los visitantes. Sin embargo, es evidente en mayor o menor medida la presencia de madera desfibrada, mayoritariamente, en elementos exteriores.

Aún cuando los revestimientos de concreto pampino de determinadas edificaciones se encuentran algo dañados y en algunos casos desprendidos, estos han evitado el incremento del deterioro en los elementos de madera.

Con relación a las edificaciones con revestimiento exteriores de madera (Fig. 1C), estos se encuentran en buen estado de conservación con mínimos grados de desfibrado.
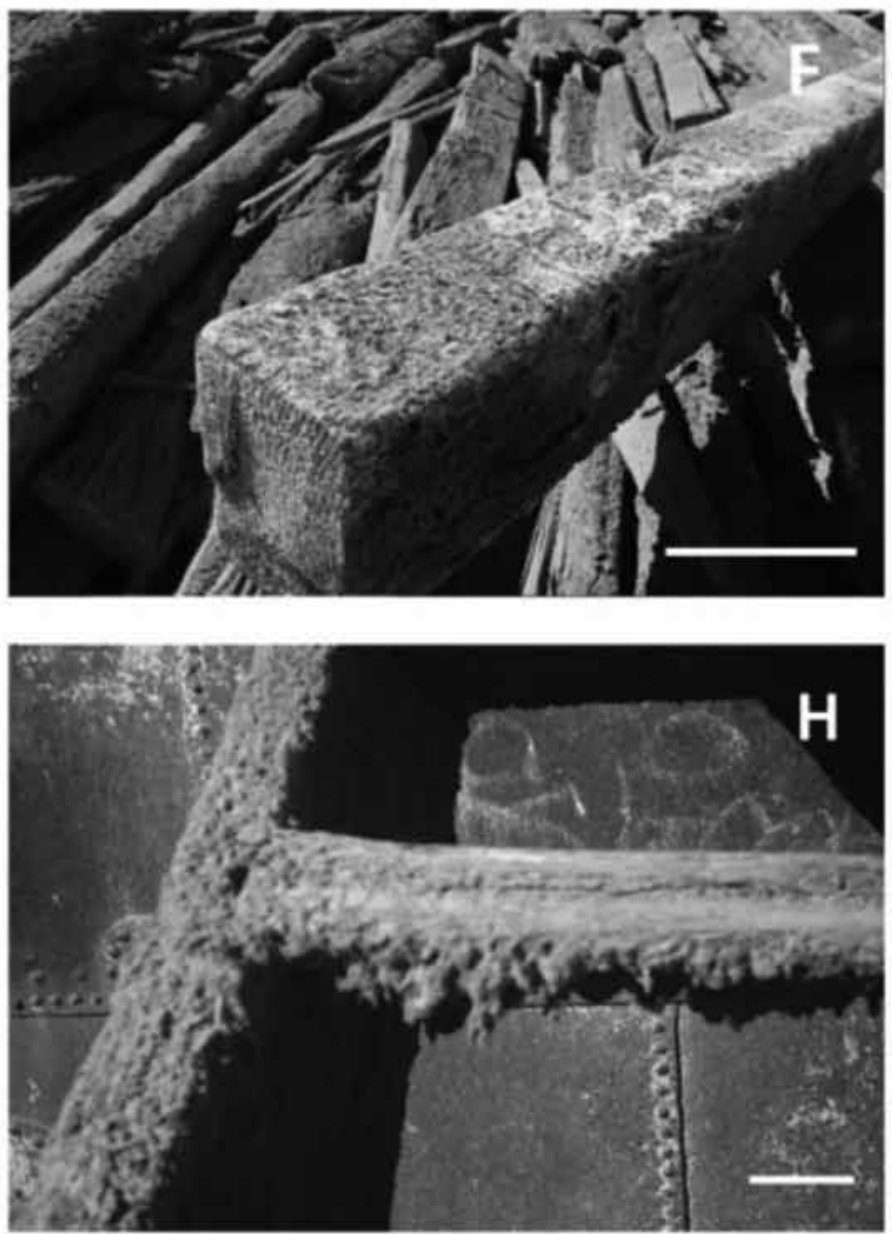

FigURA 2. Oficina salitrera Santiago Humberstone. E y F: Pilas de maderas de zona industrial. G: Reja de madera cercana a zona de lixiviación. H: Escalera cercana a zona de lixiviación. Barras en $\mathrm{E}=\mathrm{F}=10 \mathrm{~cm}, \mathrm{G}=\mathrm{H}=5 \mathrm{~cm}$. 

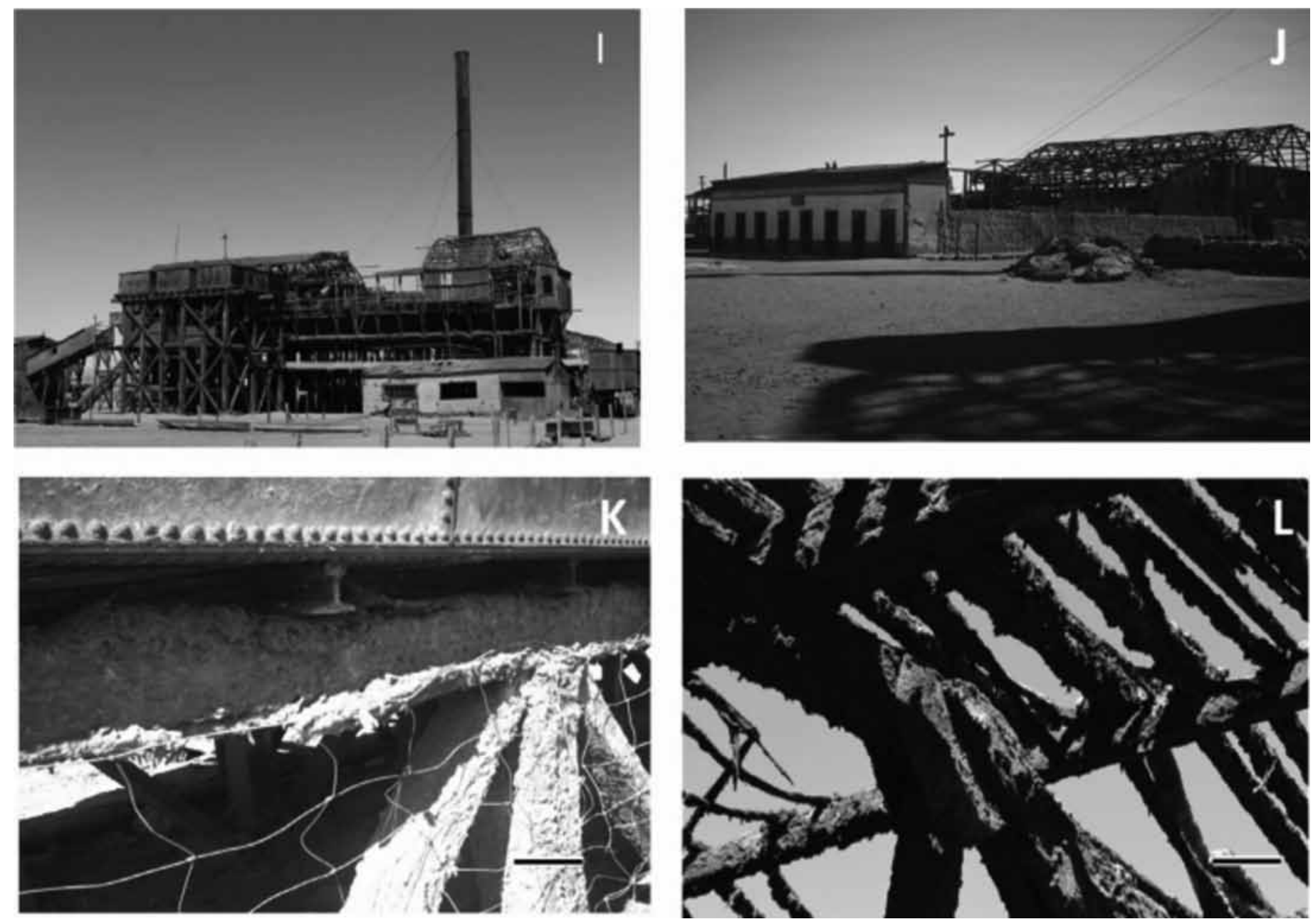

Figura 3. Oficina salitrera Santa Laura. I: Planta de lixiviación. J: Casa de yodo y SSHH públicos. K: Madera desfibrada casa de bombas. L: Madera desfibrada planta de lixiviación. Barras en $\mathrm{K}=\mathrm{L}=10 \mathrm{~cm}$.

\section{Zona Industrial}

En la zona industrial se pueden observar de manera evidente los efectos físicos del proceso de desfibrado (Figs. $2 \mathrm{E}, 2 \mathrm{~F}, 2 \mathrm{G}, 2 \mathrm{H})$, especialmente en las maderas expuestas a la intemperie, de las que se encuentran cantidades importantes desechadas en grandes montículos de material (Figs. 2E, 2F).

Así mismo es posible observar este deterioro en las maderas que constituyen la zona de talleres, bodegas, maestranzas, molienda y carpintería, entre otras. Estas maderas se encuentran en mejor estado de conservación, producto, quizás, de su menor grado de exposición: no obstante, el daño es evidente.

\section{Oficina Salitrera Santa Laura}

Zonas de edificios, espacios públicos y campamento

A diferencia de la oficina salitrera Humberstone, la oficina Santa Laura mantiene poca presencia de instalaciones pertenecientes a la zona de edificios y espacios públicos (Fig. 3J). Sin embargo, estos se mantienen en buenas condiciones con niveles variables de desfibrado en sus elementos de madera. Con respecto a la zona de campamento es posible observar vestigios de sus edificios, la que no sólo quedó expuesta a deterioros de tipo químico, físico, mecánico y biológico, sino que ha debido soportar numerosos saqueos que la han devastado en un $95 \%$ aproximada- 
mente, quedando en pie sólo parte de las vivienda de los obreros solteros y obreros casados, cuyas maderas presentan leves niveles de daño.

La zona Industrial de la oficina salitrera Santa Laura cuenta con cerca del $100 \%$ de sus instalaciones en pie (Figs. 3I, 3J, 3K, 3L), todas afectadas en menor o mayor medida por el desfibrado.

La madera de los edificios correspondientes a corral y galpón de mulas, bodega de materiales, taller de locomotoras y maestranza, compresores, enfriamiento, casa de fuerza y taller de brocas se encuentran en buen estado de conservación. Sin embargo, hay evidencia de deterioro superficial menor por desfibrado, y cuyo aumento ha sido impedido, de cierta manera, por la presencia de su envolvente metálica.
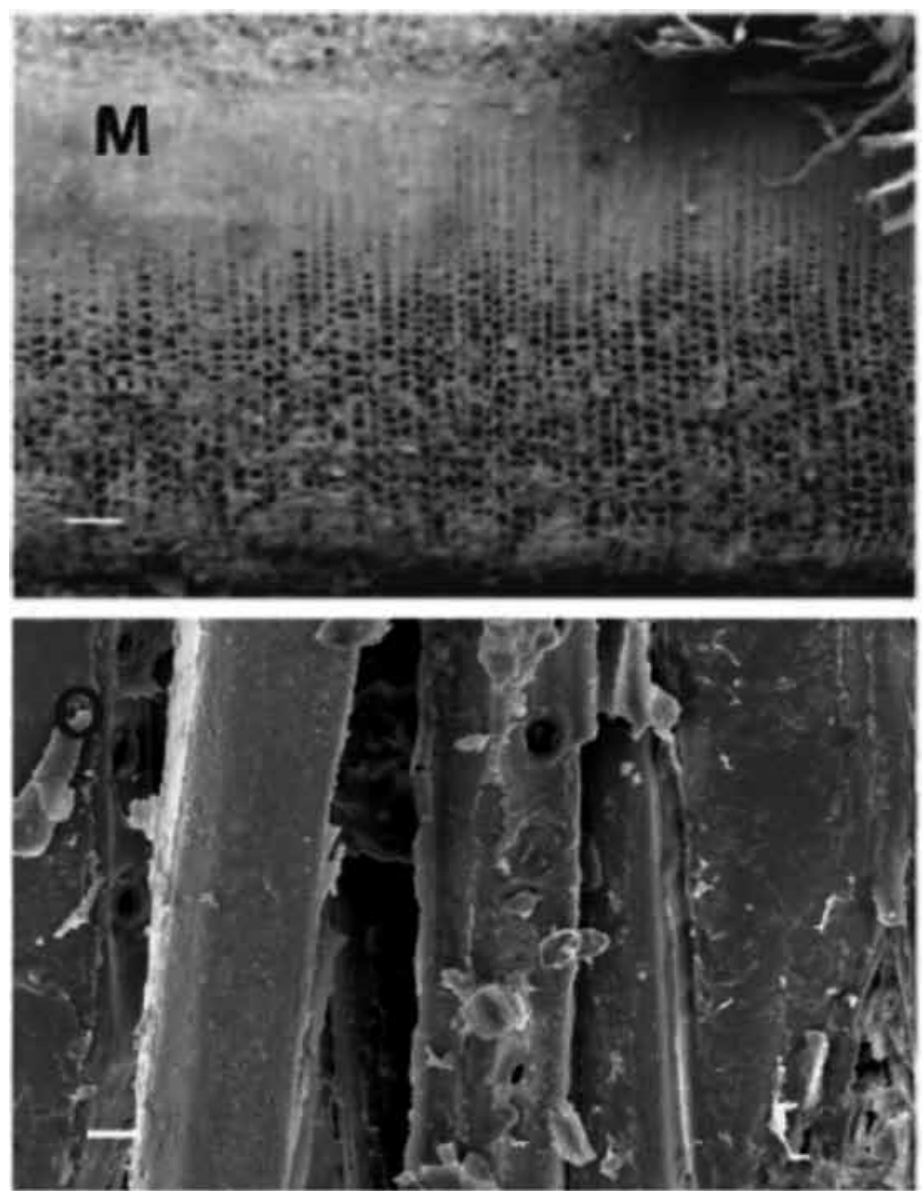

En relación con los edificios e instalaciones correspondientes a molinos primarios, correa transportadora y molinos secundarios se encuentran en buen estado. No obstante, existe un daño superficial en la mayoría de sus elementos, contrastando de forma evidente con zonas específicas de mayor profundidad de desfibrado y evidente presencia salina. De manera general, los deterioros señalados en todas las zonas descritas no presentan riesgos.

Las estructuras correspondientes a la casa de bombas, casa de yodo y planta de lixiviación (Figs. 3I, 3J, 3K, 3L) son las que presentan el mayor nivel de daño de los elementos de madera que las constituyen. Siendo esta última estructura (Fig. 3I) la que en su conjunto presenta el mayor grado de deterioro, lo que ha llevado a disminuir las secciones transversales de sus elementos. En este con-
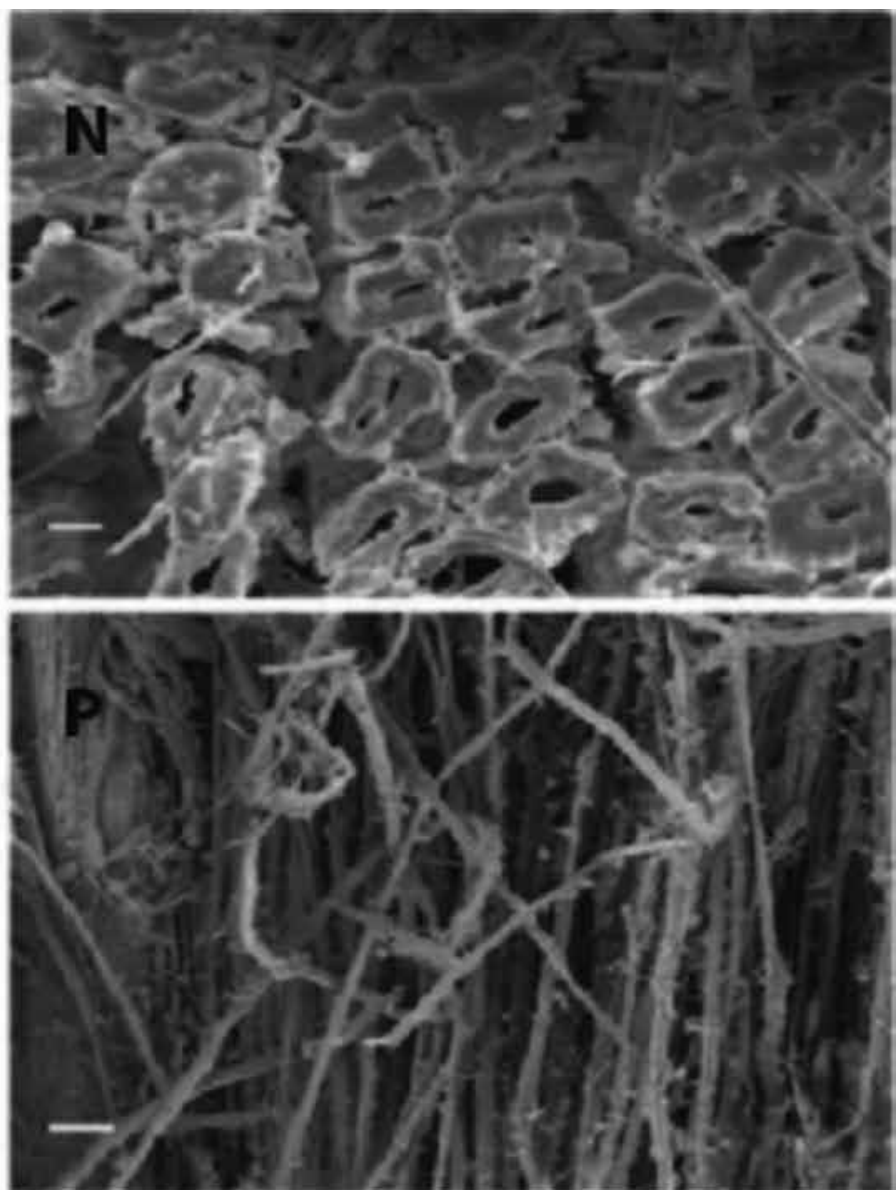

FigURa 4. Microscopía electrónica de barrido en madera de Pseudotsuga menziesii. M: Sección transversal. N: Degradación de lamela media. O y P: Plano perpendicular: fibras de celulosa. Barras en $\mathrm{M}=\mathrm{P}=200 \mu \mathrm{m}, \mathrm{N}=\mathrm{O}=20 \mu \mathrm{m}$. 
texto, los daños se presentan en aumento, desde el primer hasta el tercer nivel respectivamente, observándose importante acumulación salina en los últimos dos niveles.

\section{Análisis microscópicos}

La figura 4 muestra las imágenes de microscopía electrónica de barrido (SEM) de madera correspondiente a la especie Pseudotsuga menziesii. Deformación de la sección transversal de las fibras y su separación resultan ser evidentes (Figs. 4M, 4N, 4O), lo que también puede ser observado en los planos perpendiculares de la muestra (Fig. 4P). Estos efectos son producidos debido a la degradación de la lamela media, exponiendo con total libertad las fibras celulósicas. Similar daño se observa en la figura 5 en madera correspondiente a la especie Nothofagus obliqua. Las imágenes permiten determinar, al igual que para la especie Pseudotsuga menziesii, deformación de la sección transversal de las fibras y degradación de la lamela media (Figs. 5Q, 5R, 5S, 5T).

\section{Análisis no destructivos}

Los resultados obtenidos en los análisis no destructivos, con las maderas de Pseudotsuga menziesii obtenidas desde ambos sitios, determinaron que el valor promedio de velocidad de ultrasonido fue de $4518 \mathrm{~m} / \mathrm{s}$, velocidad no menor a $5 \%$ de lo encontrado por Niemz et al. (1994) en madera
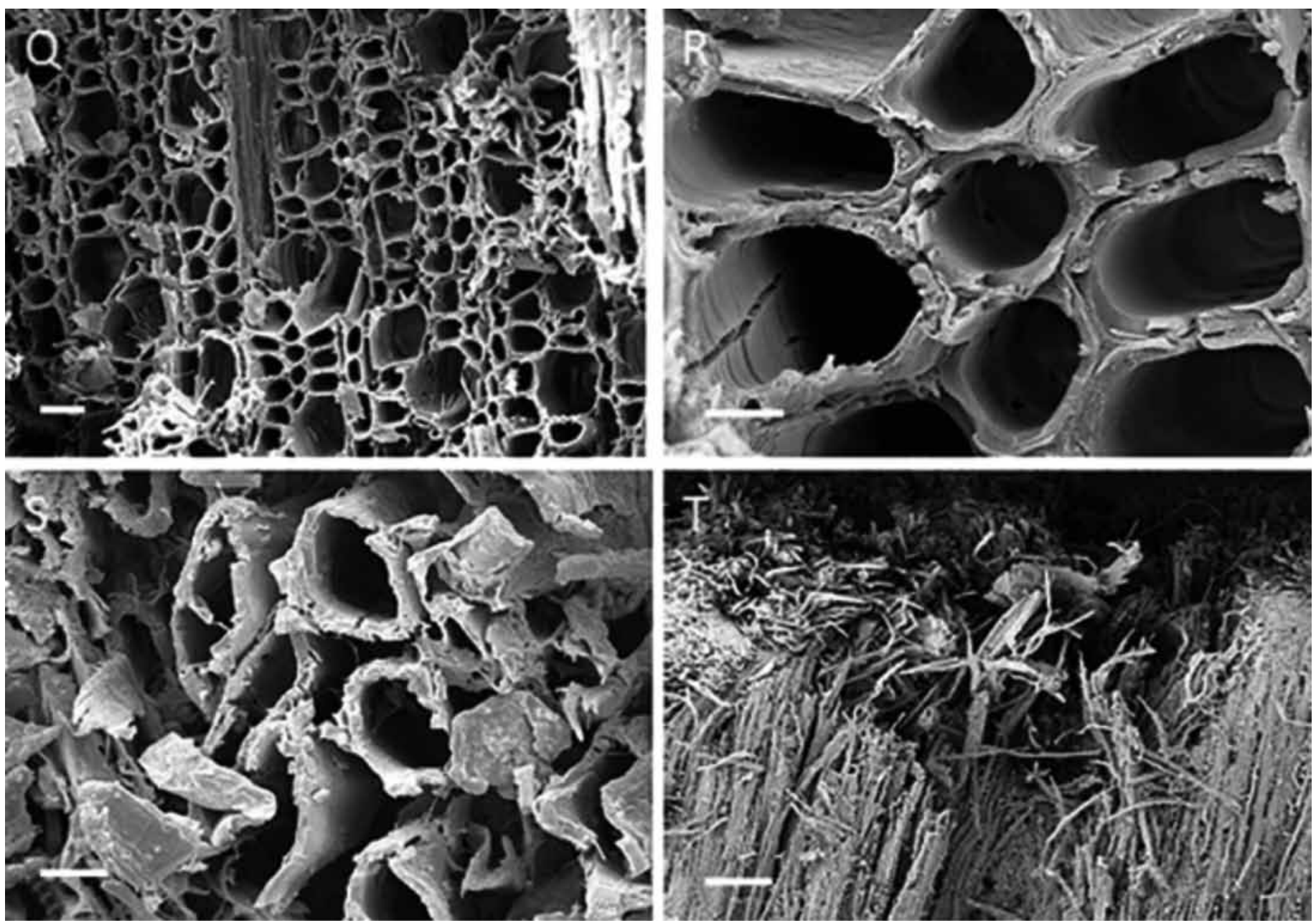

Figura 5. Microscopía electrónica de barrido en madera de Nothofagus obliqua. Q: Sección transversal. R y S: Sección transversaldegradación de lamela media. T: Sección lateral fibras de celulosa. Barras en $Q=40 \mu \mathrm{m}, \mathrm{R}=\mathrm{S}=10 \mu \mathrm{m}, \mathrm{T}=400 \mu \mathrm{m}$. 
sana. Además, a partir de las evaluaciones realizadas mediante la técnica de extracción de tornillo se obtuvo un valor de densidad promedio de $430 \mathrm{~kg} / \mathrm{m}^{3}$. Este último valor es 3,6\% menor que la densidad normal de $446 \mathrm{~kg} /$ $\mathrm{m}^{3}$, según la norma NCh $176 / 2$ of. (1986), establecida para madera sana.

\section{DISCUSIÓN}

El proceso de corrosión de la sal sobre la lamela media de la madera no ha sido comúnmente encontrado en el mundo ni ampliamente descrito en la literatura. No obstante, se ha detectado en muelles y pilares de muelles tratados con sales CCA y creosota, construcciones expuestas al mar, rutas de sal, almacenes de fertilizantes, plantas de producción de ureas (Parameswaran, 1981; Wilcox et al., 1991; Johnson et al., 1992), superficies internas de barcos, torres de refrigeración (Bootle, 2005), maderas de techumbres de zonas costeras de Sydney, Australia (Wilkins y Simpson, 1988), maderas de techumbres tratadas con retardantes al fuego basados en sulfato de amonio y fosfato de amonio (Ku erová et al., 2008) y maderas pertenecientes a las chozas de las expediciones históricas en la región del mar de Ross en la Antártica (Blanchette et al., 2002). De acuerdo con lo señalado por Blanchette et al. (2002), los antecedentes bibliográficos existentes determinan, independientemente de la fuente y composición salina, idénticos daños en los distintos elementos analizados.

Ku erová et al. (2008), a partir de estudios anatómicos de madera mediante microscopía electrónica de barrido, determinaron que tanto lignina como celulosa fueron dañadas, presentando, en algunos casos, deterioro mecánico en las fibras debido a presiones de cristalización. Asimismo estos autores agregan que la separación observada en las células se debe, aparentemente, a daños producidos en la lamela media, que es una zona altamente lignificada. Según Blanchette et al. (2002) este daño es producido por la disolución de la lamela media debido a una actividad corrosiva desarrollada por sales, siendo la separación de las fibras una forma progresiva de deterioro. En este contexto, Johnson et al. (1992) agregan que en esta actividad corrosiva los químicos penetran la madera como solutos en agua, formando cristales cuando el agua es evaporada. Con relación a las fibras celulósicas, Ku erová et al. (2008) señalan que se han observado grietas en la capa S2 de la pared celular, llegando en algunos casos a la desintegración de las fibras formando, presumiblemente, estructuras supramoleculares de celulosa. No obstante lo anterior, estos autores agregan que, de acuerdo a los estudios realizados, el daño provocado no tiene efecto en las propiedades mecánicas de las áreas adyacentes a la zona deteriorada. Lo anteriormente señalado está en concordancia con los resultados obtenidos en las evaluaciones de velocidad de ultrasonido y extracción de tornillos realizadas en el presente trabajo, los que confirman que las maderas no afectadas por el fenómeno de desfibrado mantienen sus propiedades mecánicas dentro de los valores esperados.

Con respecto al deterioro producido en las salitreras de Humberstone y Santa Laura, sin lugar a dudas, este se encuentra estrechamente ligado con el clima, los procesos industriales de fabricación del salitre y la acumulación de minerales salinos en los depósitos de nitratos chilenos, tales como: halitas, nitratos, sulfatos, yodatos, cromatos y boratos, entre otros, los que en conjunto han permitido, por muchos años, el transporte y acumulación de sales así como el posterior deterioro de las maderas constituyentes de las edificaciones. Estudios realizados por Bahamóndez y Villagrán (2009) señalan que el deterioro en las maderas fue producto de derrames de soluciones salinas en el proceso de producción del caliche, y cuya impregnación al interior de la madera junto con los constantes ciclos de secado y humidificación someterían a la madera a fuertes solicitaciones mecánicas a nivel microscópico. Estos autores agregan que daños similares, pero de menor profundidad, son atribuidos al denominado "shok térmico" debido a la exposición de algunos elementos a altas temperaturas. Blanchette et al. (2002) sostienen que los problemas de desfibrado resultan ser más serios en áreas protegidas de las lluvias lo que facilitaría la acumulación de sales. Sin embargo, para los sitios estudiados en el presente trabajo las mayores acumulaciones de sal y los mayores daños observados se encuentran al exterior y la zona geográfica 
no es afectada por lluvias. Farrell (2007) agrega que los daños pueden ocurrir rápidamente en lugares donde grandes concentraciones de sal están en contacto con madera húmeda, o muy lentamente cuando bajas concentraciones de sal son acumuladas en la madera después de la evaporación del agua. Estas situaciones fueron evidenciadas tanto en la torre de lixiviación como en los molinos primarios de la oficina salitrera de Santa Laura. De acuerdo con lo señalado por Bahamóndez y Villagrán (2009), la humedad presente se asocia con el fenómeno de camanchaca.

Como medida de preservación, es necesario detener o al menos disminuir la migración de sales al interior de las maderas considerando una remoción no abrasiva. De manera particular y urgente deben tomarse precauciones en la torre de lixiviación de Santa Laura, donde es evidente la acumulación de costras de sal en los niveles superiores, estas costras deben ser eliminadas. Al mismo tiempo es preciso profundizar en las investigaciones que han sido desarrolladas hasta el momento, Blanchette et al. (2002) coinciden en que esto es necesario con el fin de entender de mejor manera el proceso de desfibrado y así desarrollar mejores métodos de control.

\section{CONCLUSIONES}

El estudio realizado en las oficinas salitreras de Humberstone y Santa Laura ha determinado que los daños, más evidentes, son producidos por un fenómeno denominado desfibrado, que tiene como resultado la degradación de la lamela media de la madera.

El proceso de desfibrado se desarrolla desde la superficie del material, por lo que, y de acuerdo con los antecedentes bibliográficos y los resultados obtenidos mediante técnicas no destructivas, no hay disminución de la resistencia mecánica en las secciones que no han sido afectadas. Sin embargo, es necesario realizar acciones preventivas de eliminación de costras de sal en lugares donde esta se encuentre presente pues se constituyen como focos potenciales de daño acelerado.

Se deben realizar futuros trabajos con el fin de determinar mecanismos de control del deterioro, verificar el progreso o detención del daño y la eventual protección que la capa superficial de madera degradada estaría entregando al sustrato. Lo anterior se considera como información relevante en orden a aportar en el conocimiento y eventual control de esta particular forma de deterioro de la madera.

\section{RECONOCIMIENTOS}

Los autores de este trabajo agradecen la colaboración brindada por el señor Silvio Zerega Zegarra, Director Ejecutivo de la corporación Museo del Salitre, quien facilitó el ingreso a los recintos y el retiro de muestras para el desarrollo de este trabajo.

De igual manera se agradece la colaboración brindada por la Dirección de Investigación de la Universidad de Valparaíso DIUV en el financiamiento del proyecto $52 / 2011$.

\section{REFERENCIAS}

Bahamóndez, M. y A. Villagrán. 2009. Elaboración de expedientes y estudio, segunda etapa del plan de intervenciones prioritarias para las oficinas salitreras Humberstone y Santa Laura. [en línea] https://www.dropbox.com/s/58758vugpxu81qq/ Informe\%20Estudio\%20de\%20Materiales.pdf . [consulta : 07 enero 2011]

Basterra, L., L. Acuña, M. Casado, G. Ramón-Cueto y G. López. 2009. Diagnóstico y análisis de estructuras de madera mediante técnicas no destructivas: aplicación a la Plaza Mayor de Chinchón (Madrid). Informes de la Construcción 61(516):21-36.

Blanchette, R.A. y E. Simpson. 1992. Soft rot decay and wood pseudomorphs in an ancient coffin (700 BC) from tumulus MM at Gordion, Turkey. International Association of Wood Anatomists Bulletin 13:201-213.

Blanchette, R., B. Held y R. Farrell. 2002. Defibration of wood in the expedition huts of Antartica: an unusual deterioration process occurring in the polar environment. Polar Record 38(207):313-322.

Bootle, K. 2005. Wood in Australia: types, properties, and uses. McGraw Hill. Sydney, Australia. 452p. 
Culverwell, M. 2000. The mining cluster in Antofagasta. Integrating small and medium supplier into the productive chain. [en línea] http://www.aia.cl/ingles/archivos/cluster/ MalaikaCulverwell.pdf [consulta : 03 enero 2011]

Díaz-Vaz, J. 1979. Claves para la identificación de maderas de árboles nativos y cultivados en Chile. Bosque 3(1):15-25.

Farrell, R. 2007. Scientific evaluation of deterioration of historic huts of Ross Island, Antarctica. New Zealand Science Teacher 114:12-14.

Johnson, B., R. Ibach y A. Baker. 1992. Effect of salt water evaporation on tracheid separation from wood surfaces. Forest Products Journal 42(7/8):57-59.

Kučerová, I., M. Ohlídalová, J. Frankl, M. Kloiber y A. Michalcova. 2008. Defibring of historical roof beam caused by ammonium sulphate and ammonium phosphates based fire retardants. International conference on wood science for preservation of cultural heritage: Mechanical and biological factors. 5-7 Noviembre. Braga, Portugal.

Minvu. 2003. Postulación de las oficinas salitreras Humberstone y Santa Laura para su inclusión en la lista del Patrimonio Mundial/Unesco. [en línea] http://www. archivochile.com/Historia_de_Chile/sta-ma2/4/stamacult00003.pdf [consulta: 03 enero 2011]
Niemz, P., A. Aguilera y A. Rolleri. 1994. Velocidad de propagación del sonido en algunas especies creciendo en Chile y su relación con propiedades físicas. Bosque 15(2):49-54.

NCh 176/2 Of. 1986. Madera-Parte 2: Determinación de la densidad.

Parameswaran, N. 1981. Micromorphology of spruce timber after long-term service in a potash store house. Holz and Rob und Werkstoff 39:149-156.

Schneider, P., C. Freitag y J. Morrel. 1997. Decay resistance of saltwater-exposed douglas-fir piles. Wood and fiber science 29(4):370-374.

Vial, G. 1987. Panorama histórico del sector minero en Chile. Amb y Des Número especial:21-27.

Wilcox, W., E. Botsau y H, Kubler. 1991. Wood as a building material: a guide for designers and builders. 2nd Revised edition. Jhon Wiley \& Sons. Nueva York. 232p.

Wilkins, A. y A. Simpson. 1988. Defibring of roof timbers. Journal of the Institute of Wood Science 11(3):121-125.

Manuscrito recibido el 17 de junio de 2011.

Aceptado el 12 de mayo de 2014.

Este documento se debe citar como:

Ortiz, R. y R. Blanchette. 2014. Desfibrado de maderas obtenidas desde las oficinas salitreras de Humberstone y Santa Laura en Chile. Un sitio del patrimonio mundial. Madera y Bosques 20(3):59-68. 